\title{
DECAY ESTIMATES FOR THE BIHARMONIC EQUATION WITH APPLICATIONS TO SAINT-VENANT PRINCIPLES IN PLANE ELASTICITY AND STOKES FLOWS*
}

\author{
BY \\ C. O. HORGAN \\ University of Virginia, Charlottesville, Virginia
}

1. Introduction. In a recent paper [1], J. K. Knowles has established new energy decay estimates for solutions of the biharmonic equation in a semi-infinite strip, subject to nonzero boundary conditions on the near end only. Such estimates, which predict an exponential decay of energy with axial distance from the end, have been used in the analysis of Saint-Venant's principle in plane elastostatics. (See [2] for a review of recent work on principles of Saint-Venant type; for a discussion of earlier results in the linear theory of elasticity, see [3].) These results are also relevant to the study of the spatial evolution of stationary Stokes flows in a semi-infinite parallel plate channel to fully-developed Poiseuille flow [4].

Energy decay arguments involving differential inequalities have been employed previously by Knowles [5] in the analysis of Saint-Venant's principle in plane isotropic elastostatics for bounded, simply-connected domains of general shape. Similar arguments were used by Toupin [6] in his investigation of the corresponding issue for the three-dimensional elastic cylinder. In [5] an explicit estimate (lower bound) is obtained for the rate of energy decay with distance from a portion of the domain boundary carrying a self-equilibrated load. A modification of the analysis of [5] was given by Flavin [7], yielding an improved estimate of the decay rate. An alternative argument, leading to the same estimated decay rate as that obtained in [7], has been provided by Oleinik and Yosifian [8], [9]. ${ }^{1}$ The quality of the estimate for the decay rate obtained in [7-9] may be tested by comparison with the exact decay rate for the semi-infinite strip problem. ${ }^{2}$ It turns out that the results of [7-9] underestimate the exact value by a factor of nearly one-half.

In [1], a third type of argument is employed to establish energy decay for the biharmonic equation in a semi-infinite strip. The new feature contained in [1] is the consideration of a "higher-order energy" in addition to the physical energy associated with the problem. This method provides an improved estimated decay rate over that of [7-9], although still underestimating the exact value.

\footnotetext{
${ }^{*}$ Received March 30, 1988.

'See also [10], [11].

${ }^{2}$ See [1], [2] and the end of Sec. 2 of the present paper for a discussion of this exact decay rate.
}

(C)1989 Brown University 
In the present paper we describe yet another technique to examine energy decay for the biharmonic equation in a semi-infinite strip. As in [1], our approach is based on differential inequality techniques involving the higher-order energy introduced therein. The detailed arguments used here and in [1] are quite different, however. Indeed, our method is more closely akin to that of [5], [7]. A novel feature of the present work is the use of a "conservation property" for solutions of the biharmonic boundary-value problem under consideration. It turns out that the estimated decay rate we obtain, while providing an improvement over the values given in [7-9], falls short of the result obtained in [1].

It should be emphasized that the desire to obtain an estimated decay rate which closely approximates the best possible one is motivated by far more than numerical considerations. As is remarked in [1] (see also [2]), in several areas of elasticity theory where one wishes to make quantitative applications of Saint-Venant's principle, the energy decay inequality arguments have been inadequate because of the excessively conservative estimated decay rates they predict. Thus it is of interest to investigate modified energy arguments in the hope of remedying this defect.

2. The boundary-value problem. Let $R$ be the semi-infinite strip for which $0<$ $x_{1}<\infty, 0<x_{2}<h$. As in [1], we are concerned with solutions $\phi\left(x_{1}, x_{2}\right)$ of the biharmonic equation

$$
\Delta \Delta \phi \equiv \phi_{, \alpha \alpha \beta \beta}=0 \quad \text { on } R,
$$

subject to the boundary conditions

$$
\begin{aligned}
\phi\left(0, x_{2}\right) & =f\left(x_{2}\right), \quad \phi_{.1}\left(0, x_{2}\right)=g\left(x_{2}\right), \quad 0 \leq x_{2} \leq h, \\
\phi\left(x_{1}, 0\right) & =\phi_{, 2}\left(x_{1}, 0\right)=\phi\left(x_{1}, h\right)=\phi_{.2}\left(x_{1}, h\right)=0, \quad x_{1} \geq 0, \\
\phi_{, \alpha \beta}\left(x_{1}, x_{2}\right) & \left.\rightarrow 0 \text { (uniformly in } x_{2}\right) \text { as } x_{1} \rightarrow \infty,
\end{aligned}
$$

where $f$ and $g$ are prescribed functions such that

$$
f(0)=f(h)=f^{\prime}(0)=f^{\prime}(h)=g(0)=g(h)=0 .
$$

The foregoing problem arises in two different physical situations. In the theory of plane strain for a homogeneous isotropic elastic material occupying the domain $R$, with traction-free lateral sides, stresses vanishing at infinity and subject to selfequilibrated end tractions, the function $\phi$ is the Airy stress function. The stress components $\tau_{\alpha \beta}\left(x_{1}, x_{2}\right)$ in the strip are given by

$$
\tau_{\alpha \beta}=\varepsilon_{\alpha \lambda} \varepsilon_{\beta \mu} \phi_{, \lambda \mu},
$$

where $\varepsilon_{\alpha \lambda}$ is the two-dimensional alternator: $\varepsilon_{11}=\varepsilon_{22}=0, \varepsilon_{12}=-\varepsilon_{21}=1$. It is discussed in [1] (see also [2], [5]) how the boundary-value problem (2.1)-(2.5) arises naturally in the analysis of Saint-Venant's principle for linear isotropic plane elastostatics. A second physical interpretation for this problem may be given in the context of two-dimensional Stokes flows. It is shown in [4] that the entry flow problem governing the development of Stokes flows in a semi-infinite parallel plate channel to fully-developed Poiseuille flow may be described by $(2.1)-(2.5)^{3}$, where

\footnotetext{
${ }^{3}$ The asymptotic behavior at infinity (2.4) is slightly modified in the Stokes flow problem [4].
} 
now $\phi$ is the stream function with velocity components $v_{\alpha}$ given by

$$
v_{\alpha}=\varepsilon_{\alpha \beta} \phi_{, \beta} .
$$

In what follows, we shall assume the existence of a solution $\phi$ of $(2.1)-(2.4)$ which is four times continuously differentiable on the closure $\bar{R}$ of $R$. As was observed by Knowles in [1], such a smoothness assumption imposes further restrictions on the derivatives of $f$ and $g$ at $x_{2}=0, h$ in addition to those given in (2.5). Since these conditions are not used in the present paper, we shall not write them down explicitly.

A discussion is provided in [1], [2] of how an eigenfunction expansion solution of (2.1)-(2.5) in a series of eigenfunctions of the form $\phi=e^{-\lambda x_{1}} \psi\left(x_{2}\right)$ can be utilized to show that the exact rate of exponential decay of $\phi$ is given by ${ }^{4}$

$$
\operatorname{Re} \lambda \doteq 4.20 / h \text {. }
$$

3. Summary of previous and present results. The set of points $\left(x_{1}, x_{2}\right)$ in $R$ for which $x_{2}>z$ is denoted by $R_{z}$ and so $R_{0} \equiv R$. For each fixed $z>0, L_{z}$ denotes the line segment in $R$ containing the points $\left(z, x_{2}\right)$. Following [1], we call

$$
E_{1}(z)=\int_{R_{z}} \phi_{, \alpha \beta} \phi_{, \alpha \beta} d A, \quad z \geq 0,
$$

the first-order energy contained in $R_{z}$. In plane elasticity, $E_{1}(z)$ is related to the strain energy stored in $R_{z}$ ([1], [2]), while for Stokes flows, $E_{1}(z)$ is a measure of energy dissipation [4]. It has been shown by Knowles in [1] that $E_{1}(z)$ is finite.

The exponential decay estimates obtained in [5], [7-9], when specialized to the semi-infinite strip, show that

$$
E_{1}(z) \leq 2 E_{1}(0) e^{-2 k z}, \quad 0 \leq z<\infty,
$$

for some positive constant $k$. From [5], the value of $k$ is given by

$$
k=\left(\frac{\sqrt{2}-1}{2}\right)^{1 / 2} \frac{\pi}{h} \doteq \frac{1.4}{h}, \quad \text { (estimated decay rate in [5]) }
$$

while from [7-9] the improved result

$$
k=\frac{1}{\sqrt{2}} \frac{\pi}{h} \doteq \frac{2.2}{h}, \quad \text { (estimated decay rate in [7-9]) }
$$

is obtained.

Recently, Knowles [1] introduced a second-order energy $E_{2}(z)$ defined by

$$
E_{2}(z)=\int_{R_{z}} \phi_{, 1 \alpha \beta} \phi_{, 1 \alpha \beta} d A, \quad z \geq 0,
$$

(shown in [1] to be finite) and obtained the results

$$
\begin{aligned}
& E_{1}(z) \leq\left[E_{1}(0)+(1 / m) E_{2}(0)\right] e^{-2 k z}, \quad z \geq 0, \\
& E_{2}(z) \leq\left[E_{2}(0)+m E_{1}(0)\right] e^{-2 k z}, \quad z \geq 0,
\end{aligned}
$$

${ }^{4}$ The complex eigenvalues $\lambda$ are roots of the transcendental equation $\sin ^{2} \lambda-\lambda^{2}=0$. The characterization (2.8) is provided by the eigenvalue of smallest positive real part. 
for positive constants $m$ and $k$ given by

$$
m \doteq 22.4 / h^{2}, \quad k \doteq 2.7 / h .
$$

The estimated decay rate $k$ in (3.8) is an improvement over that of (3.4); however, as pointed out in [1], it still underestimates the exact result, given by $(2.8)$ :

$$
k \doteq 4.2 / h, \quad \text { (actual decay rate). }
$$

In the present paper, we also use the second-order energy $E_{2}(z)$ to obtain an estimated decay rate larger than that of (3.4). We shall show that, under the additional hypothesis that $\phi_{, 111}$ is bounded (uniformly in $x_{2}$ ) as $x_{1} \rightarrow \infty$, the estimates

$$
\begin{aligned}
& E_{1}(z) \leq 2\left[E_{1}(0)+(1 / m) E_{2}(0)\right] e^{-2 k z}, \quad z \geq 0, \\
& E_{2}(z) \leq 2\left[E_{2}(0)+m E_{1}(0)\right] e^{-2 k z}, \quad z \geq 0
\end{aligned}
$$

hold, where the positive constants $m$ and $k$ are given by

$$
m \doteq \frac{\pi^{2}}{h^{2}}(1.35) \doteq \frac{13.3}{h^{2}}, \quad k \doteq \frac{\pi(.77)}{h} \doteq \frac{2.4}{h} .
$$

We observe that the estimates (3.10) and (3.11) are less sharp than (3.6)-(3.8) obtained in [1]. As in [1], we use differential inequality techniques to establish the results (3.10) and (3.11). However, the detailed arguments are quite different and are more closely akin to those of [5], [7]. In addition, we make essential use of a "conservation property" for solutions of $(2.1)-(2.4)$, which we describe in the next section.

4. A conservation law for solutions of (2.1)-(2.4). For any solution $\phi$ of the biharmonic equation (2.1) on $R$, we have

$$
\int_{L_{z}} \phi_{, 1} \Delta \Delta \phi d x_{2}=0, \quad z \geq 0 .
$$

On integration by parts in (4.1) and making use of the boundary conditions (2.3), it follows that

$$
\int_{L_{z}}\left(\phi_{, 1} \phi_{, 1111}-2 \phi_{, 12} \phi_{, 121}+\phi_{, 22} \phi_{, 221}\right) d x_{2}=0,
$$

which we may write as

$$
\frac{d}{d z} \int_{L_{z}}\left(\phi_{, 1} \phi_{, 111}-\frac{\phi_{, .11}^{2}}{2}-\phi_{, 12}^{2}+\frac{\phi_{, 22}^{2}}{2}\right) d x_{2}=0, \quad z \geq 0 .
$$

Thus the integral

$$
\int_{L_{z}}\left(\phi_{, 1} \phi_{, 111}-\frac{\phi_{, 11}^{2}}{2}-\phi_{, 12}^{2}+\frac{\phi_{., 22}^{2}}{2}\right) d x_{2}=c=\text { constant }, \quad z \geq 0,
$$

and so is independent of $z$. The conservation law (4.4) may also be derived from considerations of invariance with respect to translations of the axial coordinate of a variational principle equivalent to the boundary-value problem under consideration. Such conservation laws, which are intimately connected with Noether's theorem, have received wide attention in elasticity theory. The relevance of such conservation 
laws to the Saint-Venant problems for a three-dimensional elastic cylinder has been discussed by Ericksen (see, e.g., [12]) and by Muncaster [13]. However, to the best of our knowledge, the only application of such conservation properties to the analysis of Saint-Venant's principle is that carried out by Horgan and Knowles [2] for the three-dimensional Neumann problem for Laplace's equation (see pp. 198-199 of [2]).

In our subsequent application of the conservation law (4.4), it is necessary to impose another hypothesis on the behavior of $\phi$ at infinity in addition to those already assumed in (2.4). We require the constant $c$ in (4.4) to be zero; in view of (2.4), a sufficient condition to ensure $c=0$ is that

$$
\phi_{, 111}=O(1) \quad \text { (uniformly in } x_{2} \text { ) as } x_{1} \rightarrow \infty
$$

which we assume henceforth. Thus (4.4) may now be written as

$$
\int_{L_{z}}\left(\phi_{, 22}^{2}-\phi_{, 11}^{2}-2 \phi_{, 12}^{2}+2 \phi_{, 1} \phi_{, 111}\right) d x_{2}=0, \quad z \geq 0 .
$$

5. Preliminary inequalities. Before proceeding to establish the results (3.10), (3.11), it is convenient to assemble here some inequalities which play a key role in our analysis.

Firstly, we rewrite (4.6) and make use of the arithmetic-geometric mean inequality in the form $-2 a b \leq \alpha a^{2}+b^{2} / \alpha(\alpha>0)$, to obtain

$$
\begin{aligned}
\int_{L_{z}}\left(\phi_{, 22}^{2}-\phi_{, 11}^{2}-2 \phi_{, 12}^{2}\right) d x_{2} & =-\int_{L_{z}} 2 \phi_{, 1} \phi_{, 111} d x_{2} \\
& \leq \int_{L_{z}}\left(\alpha \phi_{, 1}^{2}+\phi_{, 111}^{2} / \alpha\right) d x_{2} .
\end{aligned}
$$

Thus from (5.1), we have for any constant $\alpha>0$,

$$
\int_{L_{z}} \phi_{, 111}^{2} d x_{2} \geq \int_{L_{z}}\left(\alpha \phi_{, 22}^{2}-\alpha \phi_{, 11}^{2}-2 \alpha \phi_{, 12}^{2}-\alpha^{2} \phi_{, 1}^{2}\right) d x_{2}, \quad z \geq 0 .
$$

Next we recall the following well-known Wirtinger-type inequalities for sufficiently smooth functions $w\left(x_{2}\right)$ defined on $(0, h)$ :

(i) If $w\left(x_{2}\right) \in C^{1}(0, h)$ and $w(0)=0, w(h)=0$, then

$$
\int_{0}^{h} w_{, 2}^{2} d x_{2} \geq \frac{\pi^{2}}{h^{2}} \int_{0}^{h} w^{2} d x_{2}
$$

(ii) If $w\left(x_{2}\right) \in C^{2}(0, h)$ and $w(0)=w_{, 2}(0)=0, w(h)=w_{, 2}(h)=0$, then

$$
\int_{0}^{h} w_{, 22}^{2} d x_{2} \geq \frac{4 \pi^{2}}{h^{2}} \int_{0}^{h} w_{, 2}^{2} d x_{2} \text {. }
$$

(iii) If $w$ satisfies the same conditions as in (ii), then

$$
\int_{0}^{h} w_{, 22}^{2} d x_{2} \geq \frac{\mu_{0}^{4}}{h^{4}} \int_{0}^{h} w^{2} d x_{2},
$$


where $\mu_{0}$ is the smallest positive root of the transcendental equation $\cos \mu \cosh \mu=1$ and so $\mu_{0}=4.73$, a value slightly greater than $3 \pi / 2$. For simplicity, we use the latter value in (5.5) and so obtain

$$
\int_{0}^{h} w_{, 22}^{2} d x_{2}>\left(\frac{3}{2}\right)^{4} \frac{\pi^{4}}{h^{4}} \int_{0}^{h} w^{2} d x_{2}
$$

The constants appearing on the right-hand sides of the inequalities (5.3)-(5.5) are optimal and are given by smallest eigenvalues associated with a variational characterization. For proofs of (5.3) and (5.4) see [14], [15] respectively. A general class of inequalities, of which (5.5) is a special case, is considered in [16].

6. Energy decay estimates. In this section we establish the results (3.10), (3.11) for the problem $(2.1)-(2.5)$ and (4.5). As in [1], the main idea is to show that the combination $E_{2}(z)+m E_{1}(z)$ ( $m$ constant) satisfies a differential inequality. In contrast to [1], where a first-order differential inequality is obtained, we make use of a differential inequality of second-order analogous to that employed in the proof of (3.2) and (3.3) given in [5].

We first record alternative representations for the energies $E_{1}(z), E_{2}(z)$ given in (3.1) and (3.5). On using the differential equation (2.1), the divergence theorem and boundary conditions (2.3) and (2.4), it can be readily shown (see [1]) that

$$
E_{1}(z)=-\int_{L_{z}}\left(\phi_{, \alpha} \phi_{, \alpha 1}-\phi \phi_{, 111}-\phi \phi_{, 122}\right) d x_{2}, \quad z \geq 0
$$

which can be written as

$$
E_{1}(z)=-\frac{d}{d z} \int_{L_{z}}\left(\phi_{, \alpha} \phi_{, \alpha}-\phi \phi_{, 11}\right) d x_{2}, \quad z \geq 0
$$

Similarly,

$$
E_{2}(z)=-\frac{d}{d z} \int_{L_{z}} \frac{1}{2}\left(\phi_{, 11}^{2}+\phi_{, 22}^{2}\right) d x_{2}, \quad z \geq 0 .
$$

Thus, on employing the boundary conditions (2.4) at infinity, we deduce from (6.2) and (6.3) the representations

$$
\begin{array}{ll}
\int_{z}^{\infty} E_{1}(\zeta) d \zeta=\int_{L_{z}}\left(\phi_{, \alpha} \phi_{, \alpha}-\phi \phi_{, 11}\right) d x_{2}, & z \geq 0, \\
\int_{z}^{\infty} E_{2}(\zeta) d \zeta=\frac{1}{2} \int_{L_{z}}\left(\phi_{, 11}^{2}+\phi_{, 22}^{2}\right) d x_{2}, & z \geq 0 .
\end{array}
$$

From their definitions in (3.1) and (3.5), respectively, the derivatives of $E_{1}(z)$, $E_{2}(z)$ are given by

$$
\begin{aligned}
& E_{1}^{\prime}(z)=-\int_{L_{z}} \phi_{, \alpha \beta} \phi_{, \alpha \beta} d x_{2}, \quad z \geq 0, \\
& E_{2}^{\prime}(z)=-\int_{L_{z}} \phi_{, 1 \alpha \beta} \phi_{, 1 \alpha \beta} d x_{2}, \quad z \geq 0 .
\end{aligned}
$$

Let $m$ and $k$ be as yet undetermined positive constants and let

$$
F(z)=\left[E_{2}(z)+m E_{1}(z)\right]+2 k \int_{z}^{\infty}\left[E_{2}(\zeta)+m E_{1}(\zeta)\right] d \zeta, \quad z \geq 0 .
$$


Then, on using (6.4)-(6.8), we obtain the following identity, for $z \geq 0$,

$$
\begin{aligned}
F^{\prime}(z)+2 k F(z)=-\int_{L_{z}}\left[\phi_{, 1 \alpha \beta} \phi_{, 1 \alpha \beta}+m \phi_{, \alpha \beta} \phi_{, \alpha \beta}\right. \\
\left.-2 k^{2}\left(\phi_{, 11}^{2}+\phi_{, 22}^{2}+2 m \phi_{, \alpha} \phi_{, \alpha}-2 m \phi \phi_{, 11}\right)\right] d x_{2},
\end{aligned}
$$

which may be written as

$$
\begin{aligned}
F^{\prime}(z)+2 k F(z)=-\int_{L_{z}}\left[\phi_{, 111}^{2}\right. & +\phi_{, 122}^{2}+2 \phi_{, 112}^{2}+\left(m-2 k^{2}\right) \phi_{, 11}^{2} \\
& +\left(m-2 k^{2}\right) \phi_{, 22}^{2}+2 m \phi_{, 12}^{2}-4 k^{2} m \phi_{, 1}^{2}-4 k^{2} m \phi_{, 2}^{2} \\
& \left.+4 k^{2} m \phi \phi_{, 11}\right] d x_{2} \equiv-J(z) .
\end{aligned}
$$

Our objective now is to determine positive values for $k$ and $m$ for which the righthand side of $(6.10)$ is nonpositive. Suppose for the moment that such values have been found. Then it follows from (6.10) that

$$
F^{\prime}(z)+2 k F(z) \leq 0, \quad z \geq 0,
$$

which yields upon integration

$$
F(z) \leq F(0) e^{-2 k z}, \quad z \geq 0 .
$$

By virtue of the definition of $F(z)$ in (6.18), we deduce from (6.12) the inequality

$$
E_{2}(z)+m E_{1}(z) \leq F(0) e^{-2 k z}, \quad z \geq 0 .
$$

By adapting the arguments of [5] to the present case, it is easily shown that

$$
F(0) \leq 2\left[E_{2}(0)+m E_{1}(0)\right],
$$

so that (6.13) and (6.14) together yield

$$
E_{2}(z)+m E_{1}(z) \leq 2\left[E_{2}(0)+m E_{1}(0)\right] e^{-2 k z}, \quad z \geq 0 .
$$

Since $E_{1}(z), E_{2}(z)$ are nonnegative, it follows from (6.15) that

$$
\begin{aligned}
& E_{1}(z) \leq 2\left[E_{1}(0)+\frac{1}{m} E_{2}(0)\right] e^{-2 k z}, \quad z \geq 0, \\
& E_{2}(z) \leq 2\left[E_{2}(0)+m E_{1}(0)\right] e^{-2 k z}, \quad z \geq 0,
\end{aligned}
$$

which are the desired results (3.10).

It remains to verify that the choices (3.11) for $m$ and $k$ do indeed render the righthand side of (6.10) nonpositive. To this end, we show that, for the values of $m$ and $k$ given by (3.11), the integral $J(z)$ on the right-hand side of (6.10) is nonnegative. The first step is to make use of the inequality (5.2) to obtain, for any constant $\alpha>0$,

$$
\begin{aligned}
J(z) \geq \int_{L_{z}}\left[\phi_{, 122}^{2}\right. & +2 \phi_{, 112}^{2}+\left(m-2 k^{2}-\alpha\right) \phi_{, 11}^{2}+\left(m-2 k^{2}+\alpha\right) \phi_{, 22}^{2} \\
& +2(m-\alpha) \phi_{, 12}^{2}-\left(4 k^{2} m+\alpha^{2}\right) \phi_{, 1}^{2}-4 k^{2} m \phi_{, 2}^{2} \\
& \left.+4 k^{2} m \phi \phi_{, 11}\right] d x_{2} .
\end{aligned}
$$


On using the arithmetic-geometric mean inequality in the form $-2 \phi \phi_{, 11} \leq \gamma \phi^{2}+$ $\phi_{, 11}^{2} / \gamma(\gamma>0)$, we have

$$
\int_{L_{z}} 2 \phi \phi_{, 11} d x_{2} \geq-\gamma \int_{L_{z}} \phi^{2} d x_{2}-\gamma^{-1} \int_{L_{z}} \phi_{, 11}^{2} d x_{2}
$$

On employing the inequality (5.6) with the choice $w\left(x_{2}\right)=\phi\left(x_{1}, x_{2}\right)$ for each fixed $x_{1} \geq 0$ (recall the boundary conditions (2.3)), we obtain from (6.19)

$$
\int_{L_{z}} 2 \phi \phi_{, 11} d x_{2}>\frac{-\gamma h^{4}}{(3 \pi / 2)^{4}} \int_{L_{z}} \phi_{, 22}^{2} d x_{2}-\gamma^{-1} \int_{L_{z}} \phi_{, 11}^{2} d x_{2}
$$

which may be used in (6.18) to yield

$$
\begin{aligned}
J(z)>\int_{L_{z}}\left[\phi_{, 122}^{2}\right. & +2 \phi_{, 112}^{2}+\left(m-2 k^{2}-\alpha-2 k^{2} m \gamma^{-1}\right) \phi_{, 11}^{2} \\
& +\left\{m-2 k^{2}+\alpha-2 k^{2} m \gamma h^{4}(3 \pi / 2)^{-4}\right\} \phi_{, 22}^{2}+2(m-\alpha) \phi_{, 12}^{2} \\
& \left.-\left(4 k^{2} m+\alpha^{2}\right) \phi_{, 1}^{2}-4 k^{2} m \phi_{, 2}^{2}\right] d x_{2},
\end{aligned}
$$

for arbitrary positive constants $\alpha, \gamma, m$, and $k$.

The inequality (5.4), with the admissible choices $w\left(x_{2}\right)=\phi\left(x_{1}, x_{2}\right)$ for each fixed $x_{1} \geq 0$, and $w\left(x_{2}\right)=\phi, 1$ yields

$$
\int_{L_{z}} \phi_{, 2}^{2} d x_{2} \leq \frac{h^{2}}{4 \pi^{2}} \int_{L_{z}} \phi_{, 22}^{2} d x_{2}, \quad z \geq 0
$$

and

$$
\int_{L_{z}} \phi_{, 12}^{2} d x_{2} \leq \frac{h^{2}}{4 \pi^{2}} \int_{L_{z}} \phi_{, 122}^{2} d x_{2}, \quad z \geq 0,
$$

respectively. Similarly the choice $w=\phi_{, 11}$ in (5.3) yields

$$
\int_{L_{z}} \phi_{, 112}^{2} d x_{2} \geq \frac{\pi^{2}}{h^{2}} \int_{L_{z}} \phi_{, 11}^{2} d x_{2}
$$

The choice $w=\phi_{.1}$ in (5.6) gives

$$
\int_{L_{z}} \phi_{, 1}^{2} d x_{2}<\frac{h^{4}}{(3 \pi / 2)^{4}} \int_{L_{z}} \phi_{, 122}^{2} d x_{2}, \quad z \geq 0 .
$$

On using (6.22)-(6.25) in (6.21), provided that

$$
m-\alpha \leq 0 \text {, }
$$

we obtain the inequality

$$
\begin{aligned}
J(z)>\int_{L_{z}}[\{1 & \left.-\frac{\left(4 k^{2} m+\alpha^{2}\right) h^{4}}{(3 \pi / 2)^{4}}+\frac{(m-\alpha) h^{2}}{2 \pi^{2}}\right\} \phi_{, 122}^{2} \\
& +\left\{\frac{2 \pi^{2}}{h^{2}}+m-2 k^{2}-\alpha-2 k^{2} m \gamma^{-1}\right\} \phi_{, 11}^{2} \\
& \left.+\left\{m-2 k^{2}+\alpha-\frac{2 k^{2} m \gamma h^{4}}{(3 \pi / 2)^{4}}-\frac{k^{2} m h^{2}}{\pi^{2}}\right\} \phi_{, 22}^{2}\right] d x_{2} .
\end{aligned}
$$


The desired result $J(z) \geq 0$ now follows if the constants $\alpha, \gamma, m$, and $k$ can be chosen to satisfy (6.26) and to be such that the coefficients of $\phi_{, 122}^{2}, \phi_{, 11}^{2}$, and $\phi_{, 22}^{2}$ in the integrand in (6.27) are nonnegative.

It is convenient to introduce the notation

$$
m=\frac{\pi^{2}}{h^{2}} M, \quad k^{2}=\frac{\pi^{2}}{h^{2}} K^{2}, \quad \alpha=\frac{\pi^{2}}{h^{2}} \beta, \quad \gamma=\frac{\pi^{2}}{h^{2}} \delta .
$$

Thus, we seek positive constants $\beta, \delta, M$, and $K$ such that

$$
\begin{gathered}
\beta \geq M, \\
1+\frac{1}{2}(M-\beta)-\frac{\beta^{2}}{(3 / 2)^{4}}-\frac{4 k^{2} M}{(3 / 2)^{4}} \geq 0 \\
2+M-\beta-2 K^{2}\left(1+M \delta^{-1}\right) \geq 0 \\
M+\beta-K^{2}\left(M+2+\frac{2 M \delta}{(3 / 2)^{4}}\right) \geq 0 .
\end{gathered}
$$

Among all such constants satisfying the constraints (6.29)-(6.32), we desire the choice which maximizes $K$ and so, by (6.28), maximizes the estimated decay rate $k$ in (6.16) and (6.17). From (6.29), (6.30), and (6.31) it can be shown that such a value of $k$ is obtained if we choose

$$
\beta=M \text {. }
$$

Of course (6.29) is then satisfied and the remaining conditions (6.30)-(6.32) may be written as

$$
\begin{aligned}
& K^{2} \leq\left[\left(\frac{3}{2}\right)^{4}-M^{2}\right] / 4 M, \\
& K^{2} \leq\left(1+M \delta^{-1}\right)^{-1} \\
& K^{2} \leq 2 M /\left[2+M+2 M \delta\left(\frac{3}{2}\right)^{-4}\right] .
\end{aligned}
$$

It is easily verified that the right-hand sides of (6.34)-(6.36) are strictly monotone functions of each of the parameters $M$ and $\delta$. Thus an appropriate choice for $M$ and $\delta$ which maximizes the corresponding value of $K$ is obtained by equating the three expressions on the right-hand sides of (6.34)-(6.36). This leads to the following sextic polynomial equation for $M$ :

$$
\left(\frac{3}{2}\right)^{4}\left[M^{2}+4 M-\left(\frac{3}{2}\right)^{4}\right]\left[M^{3}+10 M^{2}-\left(\frac{3}{2}\right)^{4} M-2\left(\frac{3}{2}\right)^{4}\right]-2 M^{2}\left[\left(\frac{3}{2}\right)^{4}-M^{2}\right]^{2}=0,
$$

whose second ${ }^{5}$ positive root may be readily computed to be $M \doteq 1.35$. The corresponding maximum value of $K$ is then found on choosing the equality sign in (6.34). In this way, we obtain the values

$$
K=0.77, \quad M=1.35, \quad \delta=2.02 .
$$

On combining (6.38) with (6.28), we obtain the values of $m$ and $k$ stated in (3.11). This completes the proof of our main results (3.10) and (3.11).

\footnotetext{
${ }^{5}$ The smallest positive root of (6.37) gives rise to a negative value of $\delta$ and so is inadmissible.
} 
7. Stress and velocity estimates. It has been shown in [1] how the energy decay inequalities (3.6) and (3.7), with $m, k$ given by (3.8), may be used to obtain crosssectional mean-square estimates for the stresses in plane elasticity. Similar estimates follow from our results (3.10) and (3.11). Thus by (2.6) and (6.5) we have

$$
\int_{L_{z}}\left(\tau_{11}^{2}+\tau_{22}^{2}\right) d x_{2}=\int_{L_{z}}\left(\phi_{, 11}^{2}+\phi_{, 22}^{2}\right) d x_{2}=2 \int_{z}^{\infty} E_{2}(\zeta) d \zeta,
$$

and so from (3.10) it follows that

$$
\int_{L_{z}}\left(\tau_{11}^{2}+\tau_{22}^{2}\right) d x_{2} \leq \frac{2}{k}\left[E_{2}(0)+m E_{1}(0)\right] e^{-2 k z}, \quad z \geq 0,
$$

with the values of $k$ and $m$ given by (3.11). Knowles has shown in [1] that

$$
\int_{L_{z}} \phi_{, 12}^{2} d x_{2}=\int_{L_{z}} \tau_{12}^{2} d x_{2} \leq\left[E_{1}(z) E_{2}(z)\right]^{1 / 2}
$$

and so (3.10) and (3.11) yield

$$
\int_{L_{z}} \tau_{12}^{2} d x_{2} \leq \frac{2}{\sqrt{m}}\left[E_{2}(0)+m E_{1}(0)\right] e^{-2 k z}, \quad z \geq 0 .
$$

Similar cross-sectional estimates follow readily for the velocities in the Stokes flow problem. Thus, by virtue of (2.7), (6.22), (7.1), and (7.2) we obtain

$$
\int_{L_{z}} v_{1}^{2} d x_{2}=\int_{L_{z}} \phi_{, 2}^{2} d x_{2} \leq \frac{h^{2}}{2 \pi^{2} k}\left[E_{2}(0)+m E_{1}(0)\right] e^{-2 k z}, \quad z \geq 0 .
$$

The choice $w=\phi_{, 1}$ in (5.3) yields the inequality

$$
\int_{L_{z}} \phi_{, 1}^{2} d x_{2} \leq \frac{h^{2}}{\pi^{2}} \int_{L_{z}} \phi_{, 12}^{2} d x_{2}
$$

and so, by (2.7), (7.3), and (7.4) we obtain

$$
\int_{L_{z}} v_{2}^{2} d x_{2}=\int_{L_{z}} \phi_{, 1}^{2} d x_{2} \leq \frac{2 h^{2}}{\pi^{2} \sqrt{m}}\left[E_{2}(0)+m E_{1}(0)\right] e^{-2 k z}, \quad z \geq 0 .
$$

The issue of obtaining pointwise estimates is more elaborate and will not be pursued here. Interior estimates (using only $E_{1}(z)$ ) readily follow from a mean value theorem for biharmonic functions [5]. Pointwise estimates for velocities in the Stokes problem, valid up to the boundary of $R$, can be obtained from the results of the present paper for $E_{2}(z)$. As pointed out in [1], analogous results for stresses in the elasticity problem would require consideration of a third-order energy $E_{3}(z)$. Finally, upper bounds for the total energies $E_{1}(0), E_{2}(0)$ in terms of the given boundary data $f, g$ in (2.2) would be required to render the estimates (3.10) fully explicit. Such results were obtained for $E_{1}(0)$ in [5]. Modifications of the techniques used in [5] can be employed to find corresponding bounds for $E_{2}(0)$.

Acknowledgments. This work was supported by the U.S. National Science Foundation under Grant MSM-85-12825. The author is grateful to L. E. Payne of Cornell University for several helpful discussions concerning this research and for his reading of the final manuscript. Initial work on this paper was carried out during the 
summer of 1982 while the author held an appointment as visiting professor, Center for Applied Mathematics, Cornell University.

Note added in proof. Since this paper was written, a paper [17] has been published in which a generalization of the conservation law (4.4) has been established, as well as other related conservation laws for solutions of the biharmonic equation on a rectangular strip. By making use of these laws, yet another energy-type decay estimate was obtained in [17] for solutions of the biharmonic equation subject to self-equilibrated end conditions. When specialized to the semi-infinite strip, the estimated decay rate obtained in [17] is slightly less than that obtained in the present paper. In another recent paper [18], it is shown that the multiplicative factor of 2 on the right-hand side of (3.2) may be eliminated, thus repairing an obvious shortcoming in that estimate at $z=0$. By adopting the argument presented in [18], it may be easily shown that the multiplicative factors of 2 on the right-hand sides of (3.10) may also be eliminated.

\section{REFERENCES}

[1] J. K. Knowles, An energy estimate for the biharmonic equation and its application to Saint-Venant's principle in plane elastostatics, Indian J. Pure Appl. Math. 14, 791-805 (1983)

[2] C. O. Horgan and J. K. Knowles, Recent developments concerning Saint-Venant's principle, in Advances in Applied Mechanics, T. Y. Wu and J. W. Hutchinson (eds.), Vol. 23, Academic Press, San Diego, 1983, pp. 179-269

[3] M. E. Gurtin, The linear theory of elasticity, Handbuch der Physik, S. Flügge and C. Truesdell (eds.), Vol. 6a/2, Springer-Verlag, Berlin, 1972, pp. 1-295

[4] C. O. Horgan, Plane entry flows and energy estimates for the Navier-Stokes equations, Arch. Rat. Mech. Anal. 68, 359-381 (1978)

[5] J. K. Knowles, On Saint-Venant's principle in the two-dimensional linear theory of elasticity, Arch. Rat. Mech. Anal. 21, 1-22 (1966)

[6] R. A. Toupin, Saint-Venant's principle, Arch. Rat. Mech. Anal. 18, 83-96 (1965)

[7] J. N. Flavin, On Knowles' version of Saint-Venant's principle in two-dimensional elastostatics, Arch. Rat. Mech. Anal. 53, 366-375 (1974)

[8] O. A. Oleinik and G. A. Yosifian, On Saint-Venant's principle in plane elasticity theory, Dokl. Akad. Nauk. SSSR 239, 530-533 (1978) (translated in Soviet Math. Dokl. 19, 364-368 (1978))

[9] O. A. Oleinik and G. A. Yosifian, The Saint-Venant principle in the two-dimensional theory of elasticity and boundary problems for a biharmonic equation in unbounded domains, Sibirsk. Mat. Zh. 19, 11541165 (1978) (translated in Siberian Math. J. 19, 813-822 (1978))

[10] O. A. Oleinik, Energetic estimates analogous to the Saint-Venant principle and their applications, Equadiff IV, J. Fabera (ed.), Lecture Notes in Math. 703, 328-339 (1979)

[11] O. A. Oleinik, Applications of the energy estimates analogous to Saint-Venant's principle to problems of elasticity and hydrodynamics, Lecture Notes in Phys. 90, 422-432 (1979)

[12] J. L. Ericksen, Special topics in elastostatics, Advances in Applied Mechanics, C. S. Yih (ed.), Vol. 17, Academic Press, New York, 1977, pp. 189-244

[13] R. G. Muncaster, Saint-Venant's problem in nonlinear elasticity: a study of cross-sections, in Nonlinear Analysis and Mechanics: Heriot Watt Symposium, R. J. Knops (ed.), Vol. IV, Pitman, London, 1979, pp. 17-75

[14] G. H. Hardy, J. E. Littlewood, and G. Pólya, Inequalities, 2nd ed., Cambridge University Press, Cambridge, 1967

[15] S. G. Mikhlin, Variational methods in mathematical physics, Macmillan, New York, 1964

[16] C. O. Horgan, A note on a class of integral inequalities, Proc. Cambridge Philos. Soc. 74, 127-131 (1973)

[17] J. N. Flavin and R. J. Knops, Some convexity considerations for a two-dimensional traction problem, J. of Appl. Math. and Phys. (ZAMP) 39, 166-176 (1988)

[18] P. Vafeades and C. O. Horgan, Exponential decay estimates for solutions of the von Karmán equations on a semi-infinite strip, Arch. Rat. Mech. Anal. 104, 1-25 (1988) 